\title{
I/O-Efficient Computation of Water Flow Across a Terrain
}

\author{
Lars Arge* \\ MADALGO \\ University of Aarhus \\ Aarhus, Denmark \\ large@madalgo.au.dk
}

\author{
Morten Revsbæk* \\ MADALGO \\ University of Aarhus \\ Aarhus, Denmark \\ mrevs@madalgo.au.dk
}

\author{
Norbert Zeh ${ }^{\dagger}$ \\ Faculty of Computer Science \\ Dalhousie University \\ Halifax, Canada \\ nzeh@cs.dal.ca
}

\begin{abstract}
Consider rain falling at a uniform rate onto a terrain $\mathcal{T}$ represented as a triangular irregular network. Over time, water collects in the basins of $\mathcal{T}$, forming lakes that spill into adjacent basins. Our goal is to compute, for each terrain vertex, the time this vertex is flooded (covered by water). We present an I/O-efficient algorithm that solves this problem using $\mathrm{O}(\operatorname{sort}(X) \log (X / M)+\operatorname{sort}(N)) \mathrm{I} / \mathrm{Os}$, where $N$ is the number of terrain vertices, $X$ is the number of pits of the terrain, $\operatorname{sort}(N)$ is the cost of sorting $N$ data items, and $M$ is the size of the computer's main memory. Our algorithm assumes that the volumes and watersheds of the basins of $\mathcal{T}$ have been precomputed using existing methods.
\end{abstract}

\section{Categories and Subject Descriptors}

F.2.2 [Analysis of Algorithms and Problem Complexity]: Nonnumerical Algorithms and Problems-Geometrical problems and computations

\section{General Terms}

Algorithms, Theory

\section{Keywords}

Terrains, geographical information systems, I/O-efficient algorithms

\footnotetext{
*Supported in part by the Danish National Research Foundation, the Danish Strategic Research Council, and by the US Army Research office. MADALGO is the Center for Massive Data Algorithmics, a center of the Danish National Research Foundation.

${ }^{\dagger}$ Supported in part by the Natural Sciences and Engineering Research Council of Canada and the Canada Research Chairs programme. Part of this work was done while on sabbatical at MADALGO.
}

Permission to make digital or hard copies of all or part of this work for personal or classroom use is granted without fee provided that copies are not made or distributed for profit or commercial advantage and that copies bear this notice and the full citation on the first page. To copy otherwise, to republish, to post on servers or to redistribute to lists, requires prior specific permission and/or a fee.

SCG'10, June 13-16, 2010, Snowbird, Utah, USA.

Copyright 2010 ACM 978-1-4503-0016-2/10/06 ...\$10.00.

\section{INTRODUCTION}

An important problem in terrain analysis is the prediction of water flow across a terrain. Traditional approaches focus on computing the river network of the terrain under the assumption that water does not collect in the basins of the terrain. In reality, water does collect in the terrain's basins, particularly during heavy rainfall. This may cause basins to spill into adjacent basins, changing the river network of the terrain as a result. Thus, to model the flow of water across a terrain over time, it is necessary to compute the times at which the basins of the terrain spill. In this paper, we solve the more general problem of computing, for every vertex $v$ of a terrain $\mathcal{T}$, the time $t_{v}$ at which it is flooded. We assume the terrain $\mathcal{T}$ is represented as a triangular irregular network (TIN) and the amount of rain is uniform across the terrain.

The accuracy of predictions of natural phenomena, such as flooding, depends on the precision of the data used in these predictions. High-resolution elevation models even of fairly small geographic regions often exceed the size of a computer's main memory. Current GIS tools cannot handle data sets of this size efficiently and therefore need to work with models of lower resolutions. This sacrifices accuracy because lower-resolution models do not capture all terrain features. For example, in experiments we conducted to predict the flooding of coastal regions of Denmark due to rising sea levels, an elevation model with one data point every $10 \mathrm{~m}$ failed to capture a dike around an island, and the island was predicted to be flooded if the sea rises by $2 \mathrm{~m}$, while the dike can in fact withstand $2 \mathrm{~m}$ higher sea levels. Using a higherresolution model with one data point every $2 \mathrm{~m}$, we obtained the correct prediction, but storing elevation data for Denmark alone requires $500 \mathrm{~GB}$ of space at this resolution, an input size well beyond the size of main memory and beyond the reach of current GIS tools.

To process data sets beyond the size of main memory efficiently, it is necessary to develop I/O-efficient algorithms, that is, algorithms that focus on minimizing the number of disk accesses they perform to swap data between disk and internal memory, as a disk access is orders of magnitude slower than an internal-memory computation step. In this paper, we propose an I/O-efficient algorithm for computing the flooding times of all vertices of a terrain $\mathcal{T}$.

The core of the problem is computing the spill times of all basins of $\mathcal{T}$. A simple method to compute these spill times is to simulate the entire sequence of spill events of the basins of $\mathcal{T}$ and maintain the watersheds of all basins that yet have to spill, as well as predicted spill times for these basins based on their current watersheds. An internal-memory solution 
based on this idea has been presented in [14]. In Section 3.2, we discuss an $\mathrm{O}(N \log N)$ time implementation of this approach using a priority queue and a union-find structure. This simulation approach does not translate into an I/Oefficient algorithm, as the only known I/O-efficient unionfind structure [1] requires the sequence of UNION operations to be known in advance. In simulating the sequence of spill events, the set of UNION operations is known, but their order depends on the spill times of the basins. Using an internal-memory union-find structure and an I/O-efficient priority queue (e.g., $[3,12])$, a cost of $\mathrm{O}(X \alpha(X)+\operatorname{sort}(N))$ disk accesses can be achieved, where $\alpha(\cdot)$ is the inverse of Ackermann's function, $\operatorname{sort}(N) \ll N$ is the I/O complexity of sorting $N$ data items (see next section), and $X$ is the number of pits of the terrain. In contrast, the algorithm presented in this paper achieves an I/O complexity of $\mathrm{O}(\operatorname{sort}(X) \log (X / M)+\operatorname{sort}(N))$ disk accesses.

In the remainder of this section, we formally define the computational model we use, discuss previous work, and give an overview of our algorithm. Section 2 introduces the terminology and notation we use throughout the paper. Section 3 discusses our algorithm for computing the spill times of the terrain's basins and the flooding times of all terrain vertices. This algorithm makes use of an I/O-efficient meldable priority queue, which we discuss in Section 4.

\subsection{I/O Model}

We use the I/O model with one (logical) disk [2] to design and analyze our algorithm. In this model, the computer is equipped with a two-level memory hierarchy consisting of an internal memory and a (disk-based) external memory. The internal memory is capable of holding $M$ data items (vertices, edges, etc.), while the external memory is of conceptually unlimited size. All computation has to happen on data in internal memory. Data is transferred between internal and external memory in blocks of $B$ consecutive data items. Such a transfer is referred to as an $I / O$ operation or $I / O$. The cost of an algorithm is the number of I/Os it performs. The number of I/Os required to read $N$ contiguous items from disk is $\lceil N / B\rceil$. The number of I/Os required to sort $N$ items is $\operatorname{sort}(N)=\Theta\left((N / B) \log _{M / B}(N / B)\right)$ [2]. For all realistic values of $N, M$, and $B$, we have $N / B<\operatorname{sort}(N) \ll N$.

\subsection{Related Work}

Due to its importance, the problem of computing water flow across a terrain (usually in the form of a river network) has been studied extensively. Most existing methods for computing river networks assume that once water flows into a small basin of the terrain, it never flows out-in other words, basins do not spill. Therefore, to avoid water getting caught in small local basins, most flow modelling approaches first remove all basins by flooding the terrain, that is, conceptually pouring water onto the terrain until all basins are filled $[4,13,15]$. However, this often leads to unrealistic flow patterns, since many important geographical features are removed. Recent papers $[1,5]$ suggested partial flooding algorithms that flood only "small" basins, where the size of a basin can be defined using different measures, such as height, volume or area. Agarwal, Arge and Yi [1] described an $\mathrm{O}(\operatorname{sort}(N)) \mathrm{I} / \mathrm{O}$ partial flooding algorithm that removes all basins of small height. This is done by computing the topological persistence $[10,11]$ of each basin and removing the ones with persistence below a small threshold.
The key to obtaining an $\mathrm{O}(\operatorname{sort}(N)) \mathrm{I} / \mathrm{O}$ solution to this problem is an algorithm that can process a sequence of $N$ UNION and FIND operations using $\mathrm{O}(\operatorname{sort}(N))$ I/Os, assuming the entire sequence of operations is provided in advance. Arge and Revsbæk [5] extended the result of [1] to removing basins based on different geometric measures, including volume and area.

Partial flooding methods provide a basis only for approximate solutions to flow modelling, as the underlying assumption is that all "small" basins are flooded at a certain time, while all "big" basins are not. This assumption may not be true, as the spill time of a basin depends on its volume and its watershed, and the watershed of a basin may grow over time as a result of other basins spilling into it; in particular, the watershed of a big basin may grow to a size far exceeding the size of the watershed of a small basin and, thus, the big basin may spill before the small basin. To model the flow network at time $t$ accurately, it is necessary to compute the times the basins of $\mathcal{T}$ fill and remove the basins that are full at time $t$. This is much harder than the partial flooding approaches discussed above, as the above methods are based on local measures associated with each basin, while the computation of the actual spill time of each basin $\beta$ depends on the spill times of other basins that spill into $\beta$. As mentioned in the introduction, Liu and Snoeyink [14] presented an internal-memory algorithm for computing actual spill times and used it for flow prediction. While the paper does not present the algorithm in detail, we discuss an efficient implementation using a union-find structure and a priority queue in Section 3.2; this implementation is needed as part of our I/O-efficient algorithm.

We also require a tree structure that represents the nesting of the basins of $\mathcal{T}$. This tree has been termed the merge tree of $\mathcal{T}$ in $[7,8]$ (also see Section 2) and can be computed using $\mathrm{O}(\operatorname{sort}(N)) \mathrm{I} / \mathrm{Os}$ using the topological persistence algorithm of [1]. This algorithm is easily augmented to compute the lowest saddle on the boundary of each basin and, as shown in [5], the volume of each basin.

Computing the watershed sizes of all basins of $\mathcal{T}$ is harder. The watershed sizes of all basins of $\mathcal{T}$ are easily computed from the watershed sizes of all pits by summing watershed sizes bottom up in the merge tree. However, the only I/Oefficient algorithm for computing the watersheds of all pits of a terrain exactly is the one of [9]. This algorithm performs $\mathrm{O}(\operatorname{sort}(N+S)) \mathrm{I} / \mathrm{Os}$ for fat terrains, where $S$ is the size of the terrain's strip map. The size of the strip map of a fat terrain is $\Theta\left(N^{2}\right)$ in the worst case [9], in which case the exact computation of watersheds becomes infeasible. An approach often taken in practice [16-18] is to assume that water flows only along terrain edges, allowing the computation of watersheds using $\mathrm{O}(\operatorname{sort}(N))$ I/Os. While this may lead to poor approximations of the watersheds for irregular terrains [9], TIN's derived from LIDAR data, for example, are rather regular, and the computed watersheds match the real watersheds rather closely.

\subsection{New Result}

We present an algorithm that computes the flooding times of all terrain vertices using $\mathrm{O}(\operatorname{sort}(X) \log (X / M)+\operatorname{sort}(N))$ I/Os, where $N$ is the size of the terrain and $X$ is the number of pits. This assumes that the watershed sizes and volumes of all basins are given and that every vertex is labelled with the pit whose watershed contains it. The cost of computing 
this information has been discussed in the previous section. Our algorithm operates on the merge tree $\mathcal{M}$ of the given terrain $\mathcal{T}$. Given a node $\beta$ of $\mathcal{M}$, we employ a recursive strategy to compute the spill times of all basins represented by descendants of $\beta$. If there are at most $M$ such descendants, we use an augmented version of the internal-memory algorithm mentioned in the introduction to compute the spill times of their corresponding basins using $\mathrm{O}(\operatorname{sort}(Y)) \mathrm{I} / \mathrm{Os}$, where $Y$ is the number of descendants of $\beta$ plus the number of basins that spill into $\beta$. Otherwise we consider an appropriate path $P$ from $\beta$ to a descendant leaf and prove that we can compute the spill times of all basins whose parents belong to $P$ using $\mathrm{O}(\operatorname{sort}(Y)) \mathrm{I} / \mathrm{Os}$, where $Y$ is defined as above. The path $P$ is chosen so that every subtree attached to $P$ contains at most half of $\beta$ 's descendants, and we invoke our algorithm recursively on the root of each such subtree. This ensures that $\log (X / M)$ levels of recursion suffice to break $\mathcal{M}$ into subtrees of size at most $M$, to which the above internal-memory algorithm can be applied. The cost per level of recursion is $\mathrm{O}(\operatorname{sort}(X)) \mathrm{I} /$ Os. Hence, the total cost of the algorithm is $\mathrm{O}(\operatorname{sort}(X) \log (X / M)) \mathrm{I} / \mathrm{Os}$. This gives only the spill times of the basins. To compute the flooding times of all terrain vertices, we use a post-processing step that can be seen as a simpler version of the recursive step of our algorithm and performs $\mathrm{O}(\operatorname{sort}(N))$ I/Os.

The intuition behind the computation in each recursive step is the following. In general, there are two directions water can flow across any saddle of the terrain $\mathcal{T}$. Given the flow direction for each saddle, spill times could be computed rather easily, but the direction for each saddle is determined by the spill times of the two basins that merge at this saddle. For the saddles between the basins corresponding to the path $P$ in each recursive steps, we can characterize each flow direction as either "confluent" (toward the basin represented by the leaf of $P$ ) or "diffluent" (away from the leaf), and we can show that confluent spill events that influence other spill events - we call these watershed events - can themselves depend only on confluent watershed events. This allows us to perform a sweep in the confluent direction first to compute the times of all confluent watershed events. In a second phase, we sweep in the diffluent direction and use the times computed in the first phase to compute the correct times for all spill events.

\section{PRELIMINARIES}

In this section, we introduce the basic terminology used throughout this paper. In the following, we make some assumptions about the structure of the terrain that simplify the exposition in the rest of the paper. All these assumptions can be removed using standard perturbation techniques.

Terrains, pits, and basins. We consider the terrain $\mathcal{T}$ to be represented as a triangular irregular network, which is a planar triangulation each of whose vertices $v$ has an associated elevation $\mathcal{T}(v)$. The elevation of a point interior to a triangle is a linear interpolation of the elevations of the triangle vertices. In this manner, the terrain is represented as a continuous piecewise linear surface, and we use $\mathcal{T}(p)$ to refer to the elevation of the point $p \in \mathbb{R}^{2}$. We assume that no two adjacent vertices have the same elevation. A pit of $\mathcal{T}$ is a local minimum, that is, a terrain vertex all of whose neighbours have higher elevations. A saddle point of $\mathcal{T}$ is a vertex $v$ with four vertices $w_{1}, w_{2}, w_{3}, w_{4}$ among its neighbours that satisfy $\max \left(\mathcal{T}\left(w_{1}\right), \mathcal{T}\left(w_{3}\right)\right)<\mathcal{T}(v)<$ $\min \left(\mathcal{T}\left(w_{2}\right), \mathcal{T}\left(w_{4}\right)\right)$ and appear in the order $w_{1}, w_{2}, w_{3}, w_{4}$ clockwise around $v$.

A basin is a maximal connected set of points $\beta \subseteq \mathbb{R}^{3}$ such that $\mathcal{T}((x, y)) \leq z \leq h_{\beta}$, for all $(x, y, z) \in \beta$, where $h_{\beta}$ is a fixed elevation chosen as the upper boundary of $\beta$. A basin $\beta$ is maximal if there exists a saddle point $p_{\beta}$ on the boundary of $\beta$ such that $\mathcal{T}\left(p_{\beta}\right)=h_{\beta}$. The point $p_{\beta}$ is called the spill point of basin $\beta$, since water poured into $\beta$ spills over $p_{\beta}$ into an adjacent basin once $\beta$ becomes full. We assume $p_{\beta}$ is unique, that is, there are no two saddles with elevation $h_{\beta}$ on the boundary of $\beta$. We also assume exactly two basins meet in each spill point. Throughout this paper, we are interested almost exclusively in maximal basins and refer to them simply as basins. Every basin contains at least one pit, and for every pit there exists a unique (maximal) basin that contains only this pit. We call such a basin elementary.

Trickle paths, watersheds, and tributaries. The trickle path of a point $q \in \mathcal{T}$ is the path that starts at $q$, continues in the direction of steepest descent for every point it visits, and ends either in a pit $p$ or at the boundary of $\mathcal{T}$. In the former case, water falling onto $q$ collects in the elementary basin corresponding to $p$; in the latter, it flows off the edge of the terrain. The watershed of a pit $p$ is the set of points whose trickle paths end in $p$. The watershed $W_{\beta}^{0}$ of a basin $\beta$ is the union of the watersheds of all pits contained in $\beta$. More generally, we use $W_{\beta}^{t}$ to denote the watershed of basin $\beta$ at time $t$, which is the area such that water falling onto $W_{\beta}^{t}$ at time $t$ collects in basin $\beta$. A tributary of $\beta$ is a basin $\tau$ such that $\tau \cap \beta=\emptyset, \tau$ spills before $\beta$, and water falling onto $W_{\tau}^{t_{\tau}}$ at the time $t_{\tau}$ when $\tau$ spills collects in $\beta$; that is, $\tau$ spills into $\beta$ and the watershed of $\tau$ at this time becomes part of the watershed of $\beta$. Note that $\tau$ is usually a tributary of more than one basin. In particular, if $\beta$ is the smallest basin that has $\tau$ as a tributary, then $\tau$ is a tributary of every basin that contains $\beta$ but not $\tau$.

Merge tree and flow tree. The basins of $\mathcal{T}$ form a hierarchy that is easily represented using a rooted tree, the merge tree $\mathcal{M}$ of $\mathcal{T}$. The leaves of $\mathcal{M}$ are the elementary basins of $\mathcal{T}$. A basin $\beta_{1}$ is the parent of a basin $\beta_{2}$ if and only if $\beta_{2} \subset \beta_{1}$ and there exists no basin $\beta_{3}$ such that $\beta_{2} \subset$ $\beta_{3} \subset \beta_{1}$. Under the assumptions we made about $\mathcal{T}, \mathcal{M}$ is a binary tree. For a subset $S$ of nodes of $\mathcal{M}$, let $\mathcal{M}(S)$ be the subgraph of $\mathcal{M}$ induced by $S$. For a node $\alpha$ of $\mathcal{M}, \mathcal{M}_{\alpha}$ denotes the subtree of $\mathcal{M}$ induced by $\alpha$ and its descendants. We do not distinguish between merge tree nodes and their corresponding basins. For a basin $\beta$, we use $V_{\beta}$ to denote $\beta$ 's volume, $W_{\beta}^{t}$ to denote $\beta$ 's watershed at time $t$ or its size (which will be clear from the context), $E_{\beta}$ to denote the event that $\beta$ spills into an adjacent basin, and $t_{\beta}$ to denote the time of event $E_{\beta}$. We call $E_{\beta}$ the spill event associated with $\beta$. See Figure 1 for an illustration of these definitions.

Now consider a subset $S$ of nodes of $\mathcal{M}$ such that $\mathcal{M}(S)$ is a tree. The flow paths between the basins in $S$ form a flow tree $\mathcal{F}(S)$ defined as follows. Let $S^{\prime}$ be the set of leaves of $\mathcal{M}(S)$. The elements of $S^{\prime}$ are the vertices of $\mathcal{F}(S)$. There is an edge between two such vertices $\alpha_{1}$ and $\alpha_{2}$ if their watersheds touch in the common spill point of two basins $\beta_{1}, \beta_{2} \in S$. See Figure 2 .

\section{COMPUTING FLOODING TIMES}

Our algorithm for computing the flooding times of all terrain vertices has two phases. The first phase (Sections 3.13.3) computes the spill times $t_{\beta}$ of all basins of $\mathcal{T}$ using 


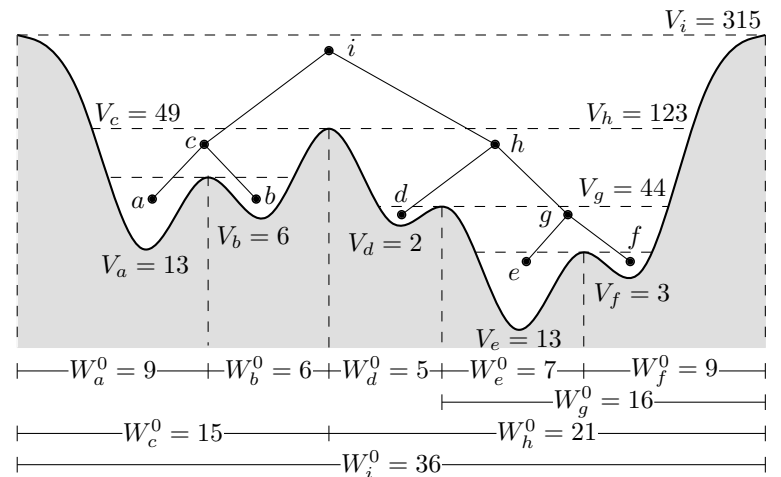

(a)

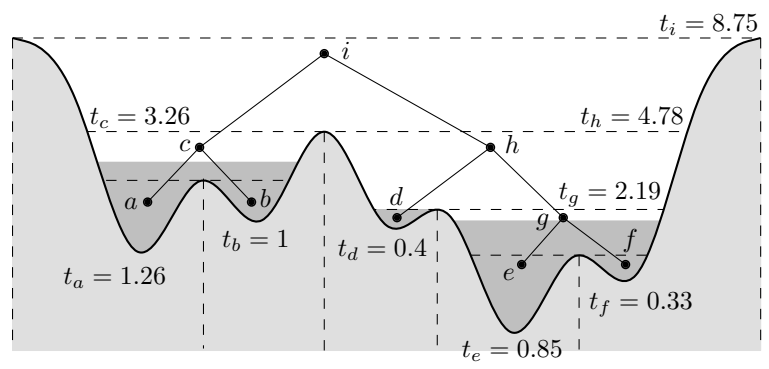

(b)

Figure 1: (a) A 1-d terrain with its corresponding merge tree and volumes and initial watersheds of its basins. (b) The spill times of the basins and the water levels in the basins at time $t=2$.

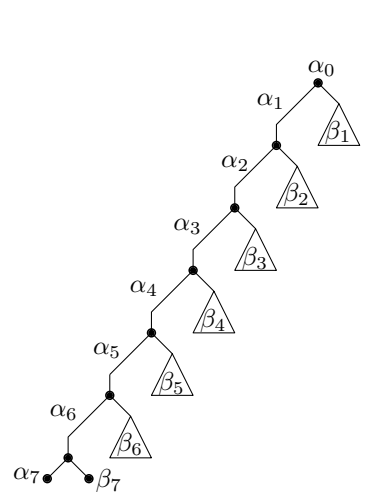

(a)

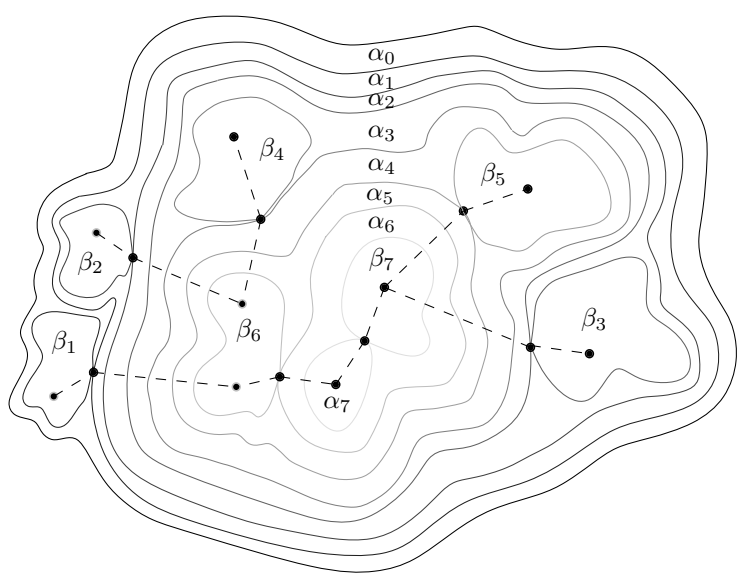

(b)

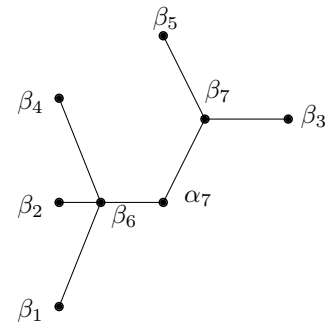

(c)

Figure 2: Figure (a) shows the merge tree $\mathcal{M}(S)$ of a set $S=\left\{\alpha_{0}, \alpha_{1}, \beta_{1}, \ldots, \alpha_{7}, \beta_{7}\right\}$ of basins. Figure (b) illustrates the nesting of these basins using contour lines through their spill points. Figure (c) shows the flow tree of basins $\alpha_{7}, \beta_{1}, \beta_{2}, \ldots, \beta_{7}$. Every edge corresponds to a spill point and joins the two basins containing the endpoints of the trickle paths starting at this spill point (shown as dashed lines in Figure (b)). Note that the trickle paths corresponding to different edges incident to a flow tree node $\beta_{i}$ may end in different pits inside the basin $\beta_{i}$. This is the case for basin $\beta_{6}$ in Figure (b).

$\mathrm{O}(\operatorname{sort}(X) \log (X / M)) \mathrm{I} /$ Os. The second phase (Section 3.4) then computes the flooding times of all vertices of $\mathcal{T}$ using $\mathrm{O}(\operatorname{sort}(N)) \mathrm{I} /$ Os.

\subsection{Computing Spill Times}

We assume the merge tree $\mathcal{M}$ is given and every node $\beta \in \mathcal{M}$ stores $V_{\beta}, W_{\beta}^{0}$, as well as identifiers of the two elementary basins $\lambda_{f}(\beta)$ and $\lambda_{r}(\beta)$ such that $\lambda_{f}(\beta) \in \mathcal{M}_{\beta}$ and $W_{\lambda_{f}(\beta)}^{0}$ and $W_{\lambda_{r}(\beta)}^{0}$ touch in $p_{\beta} ; \lambda_{f}(\beta)$ is needed for some data structure queries in our algorithm, while $\lambda_{r}(\beta)$ is the basin where water spilling from $\beta$ would collect if we poured water only into $\beta$. We further assume the elementary basins of $\mathcal{T}$ have been numbered using a preorder traversal of the flow tree $\mathcal{F}$ of $\mathcal{T}$ starting at an arbitrary root, and every elementary basin stores the preorder interval of its descendants. This information can be computed from the input of our algorithm using $\mathrm{O}(\operatorname{sort}(X))$ I/Os using standard techniques. Details appear in the full paper.
Our algorithm for computing the spill times of all basins is recursive. Every recursive call takes a node $\beta \in \mathcal{M}$ and a list $\mathcal{R}_{\beta}$ of all tributaries of $\beta$ as input. Each tributary $\tau \in \mathcal{R}_{\beta}$ stores its spill time $t_{\tau}$, its watershed $W_{\tau}:=W_{\tau}^{t_{\tau}}$ at time $t_{\tau}$, and the preorder number of an elementary basin it contains. The task of the recursive call on a node $\beta$ is to compute the spill times of all proper descendants of $\beta$ in $\mathcal{M}$. The top-level invocation takes the root $\rho$ of $\mathcal{M}$ and an empty list of tributaries as input (since $\rho$ has no tributaries). We distinguish two cases for each recursive call.

If $\left|\mathcal{M}_{\beta}\right| \leq M$, we use the algorithm in Section 3.2 below to solve the problem using $\mathrm{O}(\operatorname{sort}(X)) \mathrm{I} / \mathrm{Os}$, where $X$ is the total input size of the invocation, that is, $X:=\left|\mathcal{M}_{\beta}\right|+\left|\mathcal{R}_{\beta}\right|$.

If $\left|\mathcal{M}_{\beta}\right|>M$, we compute a heaviest path $P$ in $\mathcal{M}_{\beta}$. This path consists of a sequence of nodes $\alpha_{0}, \alpha_{1}, \ldots, \alpha_{k}$, where $\alpha_{0}=\beta, \alpha_{k}$ is a leaf, and, for $1 \leq i \leq k, \alpha_{i}$ is the child of $\alpha_{i-1}$ with the bigger subtree $\mathcal{M}_{\alpha_{i}}$ among the two children of $\alpha_{i-1}$. We use $\beta_{i}$ to denote the other child of $\alpha_{i-1}$. See 
Figure 2(a). The first step of the algorithm is to compute the spill times $t_{\alpha_{i}}$ and $t_{\beta_{i}}$ and the set $\mathcal{R}_{\beta_{i}}$ of tributaries of $\beta_{i}$, for all $1 \leq i \leq k$. To finish the computation of spill times, we recursively invoke the algorithm on each node $\beta_{i}, 1 \leq i \leq k$. As we show in Section 3.3, the computation of the spill times $t_{\alpha_{i}}$ and $t_{\beta_{i}}$, for $1 \leq i \leq k$, and of the lists of tributaries of the basins $\beta_{1}, \beta_{2}, \ldots, \beta_{k}$ takes $\mathrm{O}\left(\operatorname{sort}\left(k+\left|\mathcal{R}_{\beta}\right|\right)\right)=\mathrm{O}(\operatorname{sort}(X))$ I/Os, where $X:=\left|\mathcal{M}_{\beta}\right|+\left|\mathcal{R}_{\beta}\right|$. The path $P$ can be computed using $\mathrm{O}(\operatorname{sort}(X)) \mathrm{I} / \mathrm{Os}$ using the Euler tour technique and list ranking [6]. This gives the following result.

TheOREM 1. The spill times of all basins of a terrain $\mathcal{T}$ can be computed using $\mathrm{O}(\operatorname{sort}(X) \log (X / M)) I / O s$, where $X$ is the number of elementary basins of $\mathcal{T}$.

Proof. We prove in Section 3.2 that, given the tributaries of $\beta$, we compute the correct spill times for all basins in $\mathcal{M}_{\beta}$ in the case $\left|\mathcal{M}_{\beta}\right| \leq M$. In Section 3.3, we prove that, in the case $\left|\mathcal{M}_{\beta}\right|>M$, we compute the spill times of basins $\alpha_{i}$ and $\beta_{i}$ and the tributary lists $\mathcal{R}_{\beta_{i}}$, for $1 \leq i \leq k$, correctly. The correctness of the algorithm then follows by induction. The I/O complexity of an invocation of the algorithm with total input size $X:=\left|\mathcal{M}_{\beta}\right|+\left|\mathcal{R}_{\beta}\right|$ and merge tree size $Y:=\left|\mathcal{M}_{\beta}\right|$ is given by the recurrence

$$
T(X, Y)=\left\{\begin{array}{ll}
\mathrm{O}(\operatorname{sort}(X)) & Y \leq M \\
\mathrm{O}(\operatorname{sort}(X))+\sum_{i=1}^{k} T\left(X_{i}, Y_{i}\right) & Y>M
\end{array},\right.
$$

where $Y_{i}:=\left|\mathcal{M}_{\beta_{i}}\right|$ and $X_{i}:=Y_{i}+\left|\mathcal{R}_{\beta_{i}}\right|$. By the definition of a tributary, every basin $\tau$ with $\tau \in \mathcal{M}_{\beta}$ or $\tau \in \mathcal{R}_{\beta}$ can be the tributary of at most one basin $\beta_{i}$. Hence, we have $\sum_{i=1}^{k} X_{i} \leq X$. Furthermore, the choice of the path $P$ as a heaviest path ensures that $Y_{i} \leq Y / 2$, for all $1 \leq i \leq k$. Together, these two facts imply that the recurrence solves to $T(X, Y)=\mathrm{O}(\operatorname{sort}(X) \log (Y / M))$. For the root of $\mathcal{M}$, we have $X=Y$, that is, the overall complexity of the algorithm is $\mathrm{O}(\operatorname{sort}(X) \log (X / M))$, as claimed.

\subsection{Small Basins}

To solve the case $\left|\mathcal{M}_{\beta}\right| \leq M$, we provide an implementation of the algorithm of [14] using a union-find structure and a priority queue and extend it to take the tributaries of the basin $\beta$ into account when computing the spill times of all sub-basins of $\beta$. Before running the actual algorithm, we compute, for each tributary $\tau$ of $\beta$, the elementary sub-basin $\lambda_{r}(\tau)$ of $\beta$ that $\tau$ spills into; that is, $\tau$ spills into $\beta$ across a saddle on the boundary of $W_{\lambda_{r}(\tau)}^{0}$. These basins can be computed from the preorder numbers stored with the tributaries of $\beta$ and the preorder intervals of the elementary sub-basins of $\beta$ using $\mathrm{O}(\operatorname{sort}(X)) \mathrm{I} /$ Os. Details appear in the full paper.

The algorithm maintains a set of active basins in $\mathcal{M}_{\beta}$, which are the basins that have not spilled yet but whose children have already spilled. A basin that has spilled is finished, and a basin with at least one unfinished child is inactive. The set of active basins always contains the next basin in $\mathcal{M}_{\beta}$ to spill. We maintain the set of active basins using two data structures: a priority queue $Q$ and a unionfind structure $U$. The priority queue stores the active basins with priorities equal to their predicted spill times - these times decrease over time as we discover more tributaries of active basins. The union-find structure stores the elementary basins (leaves) in $\mathcal{M}_{\beta}$ and allows us to find, for each such basin $\alpha$, the active basin $\alpha^{\prime}$ such that $W_{\alpha}^{0}$ is currently part of $W_{\alpha^{\prime}}$. More precisely, a $\operatorname{FinD}(\alpha)$ operation returns a representative elementary sub-basin of $\alpha^{\prime}$, and it is easy to ensure that at all times, the representative of $\alpha^{\prime}$ stores the ID of $\alpha^{\prime}$. Initially, all elementary basins are active and all other basins are inactive; the predicted spill time of an elementary basin $\alpha$ is $t_{\alpha}^{\prime}:=V_{\alpha} / W_{\alpha}^{0}$. To allow the updating of predicted spill times, each active basin $\alpha$ also stores the time $u_{\alpha}$ when its watershed changed last - that is, the spill time of its most recent tributary - as well as its residual volume $V_{\alpha}^{r}$ at time $u_{\alpha}$, which is the portion of $V_{\alpha}$ left to be filled at this time. Initially, $V_{\alpha}^{r}=V_{\alpha}$ and $u_{\alpha}=0$, for every elementary basin $\alpha$ of $\mathcal{T}$.

After initializing the algorithm's data structures as just described, we process the events in $Q$ and $\mathcal{R}_{\beta}$ by increasing time until $Q$ contains only the basin $\beta$. The details of each iteration are as follows. Let $\tau$ be the next tributary in $\mathcal{R}_{\beta}$ to be processed, and let $\alpha$ be the active basin with minimum priority in $Q$. If $t_{\tau}<t_{\alpha}^{\prime}$, we remove $\tau$ from $\mathcal{R}_{\beta}$ and process $E_{\tau}$ as a watershed event with time $t_{\tau}$, as described below. If $t_{\alpha}^{\prime}<t_{\tau}$, we remove $\alpha$ from $Q$ and consider its sibling $\sigma$. If $\sigma$ is not finished, we process $E_{\alpha}$ as a watershed event with time $t_{\alpha}^{\prime}$; otherwise we process it as a basin event with time $t_{\alpha}^{\prime}$.

Watershed event: A watershed event occurs when a basin $\alpha$ spills into a non-full basin $\alpha^{\prime}$, thereby increasing the watershed of $\alpha^{\prime}$. To process a watershed event $E_{\alpha}$ with time $t$, we find the active basin $\alpha^{\prime}$ that $\alpha$ spills into using a $\operatorname{Find}\left(\lambda_{r}(\alpha)\right)$ operation on $U$. We update the information for $\alpha^{\prime}$ as $V_{\alpha^{\prime}}^{r}:=$ $V_{\alpha^{\prime}}^{r}-W_{\alpha^{\prime}}\left(t-u_{\alpha^{\prime}}\right), W_{\alpha^{\prime}}:=W_{\alpha^{\prime}}+W_{\alpha}, u_{\alpha^{\prime}}:=t$, and $t_{\alpha^{\prime}}^{\prime}:=t+V_{\alpha^{\prime}}^{r} / W_{\alpha^{\prime}}$, and then update the priority of $\alpha^{\prime}$ in $Q$ accordingly. If $\alpha \in \mathcal{R}_{\beta}$, this finishes the processing of $E_{\alpha}$. If $\alpha \in \mathcal{M}_{\beta}$, we need to update $U$ to reflect that water falling or flowing onto $W_{\alpha}$ after time $t$ flows into $\alpha^{\prime}$. We do this by performing a $\operatorname{UNION}\left(\lambda_{f}(\alpha), \lambda_{r}(\alpha)\right)$ operation on $U$ and ensuring that the representative of the resulting set of elementary basins points to $\alpha^{\prime}$. We also label the node $\alpha$ in $\mathcal{M}_{\beta}$ as finished.

Basin event: A basin event occurs when the second of two sibling basins becomes full, leaving its parent basin in $\mathcal{M}_{\beta}$ to fill. When processing a basin event $E_{\alpha}$ with time $t$, for some $\alpha \in \mathcal{M}_{\beta}$, both $\alpha$ and its sibling $\sigma$ in $\mathcal{M}_{\beta}$ are full at time $t$. Thus, we label $\alpha$ as finished in $\mathcal{M}_{\beta}$ and mark its parent $\gamma$ as active. Water that flowed into $\alpha$ before time $t$ now collects in $\gamma$. Thus, we set $W_{\gamma}:=W_{\alpha}$ and ensure that the representative of $\alpha$ now points to $\gamma$. Since both $\alpha$ and $\sigma$ are full at time $t$, we set $V_{\gamma}^{r}:=V_{\gamma}-V_{\alpha}-V_{\sigma}, u_{\gamma}:=t$, and $t_{\gamma}^{\prime}:=t+V_{\gamma}^{r} / W_{\gamma}$. Then we insert $\gamma$ into $Q$ with priority $t_{\gamma}^{\prime}$.

The following lemma states the correctness and I/O complexity of the above procedure.

Lemma 1. Let $\beta$ be a basin with at most $M$ sub-basins, and let $X:=\left|\mathcal{M}_{\beta}\right|+\left|\mathcal{R}_{\beta}\right|$. The spill times of all basins $\alpha \in \mathcal{M}_{\beta}$ can be computed using $\mathrm{O}(\operatorname{sort}(X)) I / O s$.

Proof. To bound the I/O complexity of the procedure, we observe that $Q, U$, and $\mathcal{M}_{\beta}$ fit in memory and that scanning the sorted list of tributaries $\mathcal{R}_{\beta}$ requires us to keep only one buffer block of size $B$ in memory. Thus, apart from sorting the tributaries in $\mathcal{R}_{\beta}$ by their spill times using $\mathrm{O}(\operatorname{sort}(X)) \mathrm{I} / \mathrm{Os}$, the algorithm only loads $\mathcal{M}_{\beta}$ into memory and scans $\mathcal{R}_{\beta}$, which takes $\mathrm{O}(X / B)$ I/Os. All other processing happens in memory. 
To prove the correctness of the algorithm, we consider the sequence of events $E_{1}, E_{2}, \ldots, E_{X}$ affecting basin $\beta$ and its sub-basins, sorted by their times $t_{1}, t_{2}, \ldots, t_{X}$. That is, every event $E_{i}$ is an event $E_{\alpha}$ with $\alpha \in \mathcal{M}_{\beta}$ or such that $\alpha \notin \mathcal{M}_{\beta}$ and $\alpha$ is a tributary of a basin $\alpha^{\prime} \in \mathcal{M}_{\beta}$, in which case $\alpha$ is also a tributary of $\beta$ and belongs to $\mathcal{R}_{\beta}$.

We use $t_{i}^{\prime}$ to denote the "current" predicted time of the event $E_{i}$. Consider the basin $\alpha$ such that $E_{i}=E_{\alpha}$. Then we define $t_{i}^{\prime}:=t_{i}$ if $\alpha$ is a tributary of $\beta$ and $t_{i}^{\prime}:=\infty$ if $\alpha \in \mathcal{M}_{\beta}$ and $\alpha$ is inactive; if $\alpha \in \mathcal{M}_{\beta}$ and $\alpha$ is active, we define $t_{i}^{\prime}$ to be its priority in $Q$. We use induction on $i$ to prove that $t_{i}^{\prime}=t_{i}$ when event $E_{i}$ is processed. To do so, we prove that the following holds after processing event $E_{i}$ :

(i) Events $E_{1}, E_{2}, \ldots, E_{i}$ have been processed. No other events have been processed.

(ii) $W_{\alpha}=W_{\alpha}^{t_{i}}$, for all active basins $\alpha \in \mathcal{M}_{\beta}$.

(iii) $t_{i+1}^{\prime}=t_{i+1}$, while $t_{j}^{\prime} \geq t_{j}$, for all $j>i+1$.

The base case is $i=0$, setting $t_{0}=0$. Property (i) holds because no events have been processed yet. Property (ii) holds because initially $W_{\alpha}=W_{\alpha}^{0}$, for every elementary basin in $\mathcal{M}_{\beta}$, which is exactly the set of active basins at the beginning of the procedure. To see that (iii) holds, observe that $t_{j}^{\prime}=t_{j}$ if $E_{j}=E_{\tau}$, for some tributary $\tau$ of $\beta$. If $E_{j}=E_{\alpha}$, for $\alpha \in \mathcal{M}_{\beta}$, and $\alpha$ is inactive, then $t_{j}^{\prime}=\infty$; if $\alpha$ is active, then $t_{j}^{\prime}=V_{\alpha} / W_{\alpha}^{0} \geq t_{j}$. Moreover, if $E_{1}=E_{\alpha}$, for some $\alpha \in \mathcal{M}_{\beta}$, then $\alpha$ has no tributaries and $t_{1}=V_{\alpha} / W_{\alpha}^{0}=t_{1}^{\prime}$.

For the inductive step, consider $i>0$ and assume the claim holds for all $j<i$. By part (i) of the inductive hypothesis, $E_{i-1}$ is the $(i-1)$ st event to be processed. By part (iii) of the inductive hypothesis, we have $t_{i}^{\prime}=t_{i}$ and $t_{j}^{\prime} \geq t_{j}>t_{i}$, for all $j>i$, after processing $E_{i-1}$. Hence, $E_{i}$ is the $i$ th event to be processed. This establishes (i).

To prove (ii), we consider the two possible types of event $E_{i}$ separately. Let $E_{i}=E_{\alpha}$. If $E_{i}$ is a watershed event, part (ii) of the inductive hypothesis implies that we identify the correct watershed $W_{\alpha^{\prime}}$ into which $\alpha$ spills at time $t_{i}$ and, hence, that we update $W_{\alpha^{\prime}}$ to $W_{\alpha^{\prime}}^{t_{i}}$; all other watersheds remain unchanged. If $E_{i}$ is a basin event, $\alpha$ and its sibling are full at time $t_{i}$, while $\alpha$ 's parent $\gamma$ has to fill. Furthermore, all water flowing into $\gamma$ immediately before time $t_{i}$ collects in $\alpha$ because $\alpha$ 's sibling is already full. Therefore, $W_{\gamma}^{t_{i}}=W_{\alpha}^{t_{i}}$, and we correctly set $W_{\gamma}:=W_{\alpha}$.

Finally, consider (iii). We consider only events $E_{j}=E_{\alpha}$ with $\alpha$ an active basin in $\mathcal{M}_{\beta}$ because, by definition, $t_{j}^{\prime}=t_{j}$, for every spill event $E_{j}$ of a tributary of $\beta$, and $t_{j}^{\prime}=\infty$, for every spill event $E_{j}$ of an inactive basin in $\mathcal{M}_{\beta}$. It is easily verified that we update $t_{\alpha}^{\prime}$ correctly whenever we increase $W_{\alpha}$. By (ii), each event $E_{h}$ with $h \leq i$ updates $W_{\alpha}$ if and only if $E_{h}=E_{\tau}$, for some tributary $\tau$ of $\alpha$. Thus, $t_{j}^{\prime} \geq t_{j}$, for all $j \geq i$. For $j=i+1$, the spill events of the tributaries of $\alpha$ are a subset of $E_{1}, E_{2}, \ldots, E_{i}$, as they have to happen before $E_{\alpha}=E_{i+1}$. Thus, we have $t_{i+1}^{\prime}=t_{i+1}$ after $E_{i}$ is processed.

\subsection{Basin Sets With Linear Merge Trees}

Now consider a path $P=\left\langle\beta=\alpha_{0}, \alpha_{1}, \ldots, \alpha_{k}\right\rangle$ from a merge tree node $\beta$ to a descendant leaf $\alpha_{k}$, and let $\beta_{i}$ be the sibling of $\alpha_{i}$, for all $1 \leq i \leq k$. The basic step of our solution for the case $\left|\mathcal{M}_{\beta}\right|>M$ computes the spill times of $\alpha_{1}, \beta_{1}, \alpha_{2}, \beta_{2}, \ldots, \alpha_{k}, \beta_{k}$, as well as the lists of tributaries
$\mathcal{R}_{\beta_{1}}, \mathcal{R}_{\beta_{2}}, \ldots, \mathcal{R}_{\beta_{k}}$ of the basins $\beta_{1}, \beta_{2}, \ldots, \beta_{k}$. Our algorithm for this problem operates on the flow tree $\mathcal{F}:=\mathcal{F}(S)$ of the set $S$ of basins in $P$. Note that every edge in $\mathcal{F}$ corresponds to the spill point connecting two basins $\alpha_{i}$ and $\beta_{i}$, for $1 \leq i \leq k$, and that $\lambda_{f}\left(\alpha_{i}\right)=\lambda_{r}\left(\beta_{i}\right)$ and $\lambda_{f}\left(\beta_{i}\right)=\lambda_{r}\left(\alpha_{i}\right)$ in this case. So the tree is easy to construct using a constant number of sorting and scanning steps of $S$. Details appear in the full paper.

We call a spill event $E_{\beta_{i}}$ confluent, as the water spilling from $\beta_{i}$ at time $t_{\beta_{i}}$ spills towards $\alpha_{k}$. Similarly, we call a spill event $E_{\alpha_{i}}$ diffluent, as the water spilling from $\alpha_{i}$ at time $t_{\alpha_{i}}$ spills away from $\alpha_{k}$ ( $\alpha_{k}$ is a sub-basin of $\left.\alpha_{i}\right)$. We define $\mathcal{F}_{\beta_{i}}$ to be the subtree of $\mathcal{F}$ rooted in $\beta_{i}$, assuming $\alpha_{k}$ is chosen as the root of $\mathcal{F}$. Our algorithm proceeds in two phases. The confluent phase computes times $t_{\beta_{1}}^{\prime}, t_{\beta_{2}}^{\prime}, \ldots, t_{\beta_{k}}^{\prime}$. These times satisfy $t_{\beta_{i}}^{\prime}=t_{\beta_{i}}$ if $E_{\beta_{i}}$ is a watershed event and $t_{\beta_{i}}^{\prime} \geq t_{\beta_{i}}$ if $E_{\beta_{i}}$ is a basin event. In addition, this phase constructs a list $L_{\beta_{i}}$, for each $1 \leq i \leq k$, which is a superset of those tributaries of $\beta_{i}$ that belong to $\mathcal{F}_{\beta_{i}}$ or spill into $\beta$ across a saddle on the boundary of a basin $\beta_{j} \in \mathcal{F}_{\beta_{i}}$. The second, diffluent phase computes times $t_{\alpha_{1}}^{\prime \prime}, t_{\alpha_{2}}^{\prime \prime}, \ldots, t_{\alpha_{k}}^{\prime \prime}$ and $t_{\beta_{1}}^{\prime \prime}, t_{\beta_{2}}^{\prime \prime}, \ldots, t_{\beta_{k}}^{\prime \prime}$ from the times and potential tributary lists computed in the confluent phase, and we prove that $t_{\alpha_{i}}^{\prime \prime}=t_{\alpha_{i}}$ and $t_{\beta_{i}}^{\prime \prime}=t_{\beta_{i}}$, for all $1 \leq i \leq k$. In the process, the diffluent phase computes the list $\mathcal{R}_{\beta_{i}}$ of tributaries of $\beta_{i}$, for every $1 \leq i \leq k$.

Intuitively, this two-phase approach works because the confluent phase ensures that the spill times of all basins $\beta_{i}$ that are tributaries of other basins are computed correctly (since $\beta_{i}$ can be a tributary only if its spill event is a watershed event). A basin $\alpha_{i}$ can have only confluent tributaries, since basins $\alpha_{1}, \alpha_{2}, \ldots, \alpha_{k}$ are nested. A basin $\beta_{i}$ can have only one diffluent tributary, namely $\alpha_{i}$, because $\beta_{i}$ is a subbasin of $\alpha_{j}$, for all $j<i$, and $\alpha_{i}$ is a super-basin of $\alpha_{h}$, for all $h>i$. Thus, by processing the basins "outwards" from $\alpha_{k}$ that is, by decreasing index $i$-in the diffluent phase, we can ensure that the spill times of all tributaries of a basin are known by the time the basin is processed.

\subsubsection{From Tributaries to Spill Times}

Both phases of our algorithm use the same elementary procedure to compute a predicted spill time for a basin. To avoid duplication, we describe this procedure here and refer to it as procedure FindSPILLTime later. The input of this procedure is a basin $\alpha$, a time $u_{\alpha}$, the residual volume $V_{\alpha}^{r}$ of $\alpha$ at time $u_{\alpha}$, and the watershed $W_{\alpha}=W_{\alpha}^{u_{\alpha}}$ of $\alpha$ at time $u_{\alpha}$. In addition, we are given a priority queue $Q$ containing a set of potential tributaries of $\alpha$; each entry $\tau \in Q$ satisfies $t_{\tau}^{\prime}>u_{\alpha}$, where $t_{\tau}^{\prime}$ denotes the priority of $\tau$. The output of the procedure is a predicted spill time $t_{\alpha}^{\prime}$ of $\alpha$ and a list $L$ of entries removed from $Q$ while computing $t_{\alpha}^{\prime}$.

Initially, we set $t_{\alpha}^{\prime}:=u_{\alpha}+V_{\alpha}^{r} / W_{\alpha}$. Then we repeat the following steps to update $t_{\alpha}^{\prime}$ until either $Q$ is empty or the minimum entry $\tau$ in $Q$ satisfies $t_{\tau}^{\prime} \geq t_{\alpha}^{\prime}$. We remove the minimum entry $\tau$ (which satisfies $t_{\tau}^{\prime}<t_{\alpha}^{\prime}$ ) from $Q$ and append it to $L$. Then we set $V_{\alpha}^{r}:=V_{\alpha}^{r}-W_{\alpha}\left(t_{\tau}^{\prime}-u_{\alpha}\right), W_{\alpha}:=W_{\alpha}+W_{\tau}$, $u_{\alpha}:=t_{\tau}^{\prime}$, and $t_{\alpha}^{\prime}:=u_{\alpha}+V_{\alpha}^{r} / W_{\alpha}$.

Lemma 2. Assume at the beginning of procedure FinDSpillTime, $W_{\alpha}$ and $V_{\alpha}^{r}$ reflect the actual watershed and residual volume of $\alpha$ at time $u_{\alpha}, Q$ contains only entries $\tau$ with $t_{\tau}^{\prime} \geq u_{\alpha}$, and $t_{\tau}^{\prime}<t_{\alpha}$ if and only if $\tau$ is a tributary of $\alpha$. Assume further that every tributary $\tau \in Q$ of $\alpha$ satisfies $t_{\tau}^{\prime}=t_{\tau}$. Then $t_{\alpha}^{\prime} \geq t_{\alpha}$ when the procedure terminates. If $Q$ 
contains every tributary $\tau$ of $\alpha$ with $t_{\tau} \geq u_{\alpha}$, then $t_{\alpha}^{\prime}=t_{\alpha}$, and $L=\left\{\tau \in \mathcal{R}_{\alpha} \mid t_{\tau} \geq u_{\alpha}\right\}$.

Proof. First assume for the sake of contradiction that $t_{\alpha}^{\prime}<t_{\alpha}$ when the algorithm terminates. Then the last element $\tau \in Q$ processed by procedure FindSpILLTime satisfies $t_{\tau}^{\prime}<t_{\alpha}^{\prime}$, as the update of $t_{\alpha}^{\prime}$ when processing an entry $\tau$ does not decrease $t_{\alpha}^{\prime}$ below $t_{\tau}^{\prime}$. Hence, every processed entry $\tau$ satisfies $t_{\tau}^{\prime}<t_{\alpha}$. This implies that all processed elements are tributaries of $\alpha$ that satisfy $u_{\alpha}<t_{\tau}^{\prime}=t_{\tau}<t_{\alpha}$, and we process them in order. Thus, after processing every tributary $\tau$, we have $W_{\alpha} \leq W_{\alpha}^{t_{\tau}}$, which implies that $t_{\alpha}^{\prime} \geq t_{\alpha}$, a contradiction.

Now assume $Q$ contains all tributaries of $\alpha$ and $t_{\alpha}^{\prime}>t_{\alpha}$ when procedure FindSPILLTime finishes. This is possible only if we do not process all tributaries of $\alpha$. An unprocessed tributary $\tau$ would have to remain in $Q$ by the end of the procedure and, thus, satisfies $t_{\tau}^{\prime}>t_{\alpha}^{\prime}$. However, $t_{\tau}^{\prime}=t_{\tau}<$ $t_{\alpha}<t_{\alpha}^{\prime}$, again a contradiction.

Finally, observe that, if $t_{\alpha}^{\prime}=t_{\alpha}$, we process exactly the elements $\tau \in Q$ with $t_{\tau}^{\prime}<t_{\alpha}$, and place them into $L$. These are exactly the tributaries of $\alpha$ that satisfy $t_{\tau} \geq u_{\alpha}$.

\subsubsection{Confluent Phase}

To implement the confluent phase of the algorithm, we root the flow tree $\mathcal{F}$ in $\alpha_{k}$ and process its nodes in postorder. With every node $\alpha \in \mathcal{F}$ we associate a list $\mathcal{S}_{\alpha} \subseteq \mathcal{R}_{\beta}$ containing all tributaries $\tau$ of $\beta$ that spill into $\beta$ across a saddle on the boundary of $W_{\alpha}^{0}$. These lists are easy to compute using $\mathrm{O}(\operatorname{sort}(X)) \mathrm{I} / \mathrm{Os}$ from the preorder numbers of the elementary basins associated with the tributaries, assuming that every basin in $\mathcal{M}$ stores the largest preorder interval of all its elementary sub-basins, which is easily computed in a preprocessing step using a bottom-up traversal in $\mathcal{M}$. Details appear in the full paper.

During the traversal of $\mathcal{F}$, we ensure that visiting a node $\beta_{i}$ produces a priority queue $Q_{\beta_{i}}$ that contains all basins $\tau$ that satisfy (i) $\tau \in\left\{\beta_{j}\right\} \cup \mathcal{S}_{\beta_{j}}$, for some $\beta_{j} \in \mathcal{F}_{\beta_{i}}$, and (ii) $t_{\tau}^{\prime} \geq t_{\beta_{h}}^{\prime}$, for all $\beta_{h}$ on the path from $\beta_{j}$ to $\beta_{i}$ in $\mathcal{F}$, inclusive. At any point during the postorder traversal, there is a set of active nodes, which are nodes that have been visited already but whose parents in $\mathcal{F}$ have not. We maintain the priority queues of all active nodes in a sequence sorted in the order these nodes are visited and represent this sequence of priority queues using a data structure that supports INSERT and DELETEMIN operations on the last priority queue in the sequence, the creation of a new priority queue at the end of the sequence, as well as a MELD operation, which replaces the last two priority queues in the sequence with their union. In Section 4, we show that the external heap of Fadel et al. [12] can be extended to process a sequence of $N$ Insert, Deletemin, Create, and Meld operations using $\mathrm{O}(\operatorname{sort}(N)) \mathrm{I} / \mathrm{Os}$.

The processing of a node $\beta_{i}$ distinguishes two cases. If $\beta_{i}$ is a leaf, we create a new priority queue $Q_{\beta_{i}}$ at the end of the sequence of priority queues of active nodes. If $\beta_{i}$ is an internal node with children $\beta_{j_{1}}, \beta_{j_{2}}, \ldots, \beta_{j_{h}}$, the corresponding priority queues $Q_{\beta_{j_{1}}}, Q_{\beta_{j_{2}}}, \ldots, Q_{\beta_{j_{h}}}$ are at the end of the current sequence of priority queues, and we construct $Q_{\beta_{i}}$ by melding these priority queues. In both cases, we continue by inserting all elements $\tau \in \mathcal{S}_{\beta_{i}}$ into $Q_{\beta_{i}}$, with priority $t_{\tau}^{\prime}=t_{\tau}$. Then we use procedure FindSPILLTIME to compute $t_{\beta_{i}}^{\prime}$ and $L_{\beta_{i}}$; the input to the procedure is $\beta_{i}, Q_{\beta_{i}}$, $u_{\beta_{i}}:=0, W_{\beta_{i}}:=W_{\beta_{i}}^{0}$, and $V_{\beta_{i}}^{r}:=V_{\beta_{i}}$. Once this procedure terminates, we insert $\beta_{i}$ into $Q_{\beta_{i}}$, with priority $t_{\beta_{i}}^{\prime}$.

The confluent phase terminates when the traversal reaches the root $\alpha_{k}$ of $\mathcal{F}$. Since $E_{\alpha_{k}}$ is a diffluent spill event, we do not compute its time in this phase of the algorithm. We only construct a list $L_{\alpha_{k}}$ by collecting all elements in $Q_{\beta_{j_{1}}}, Q_{\beta_{j_{2}}}, \ldots, Q_{\beta_{j_{h}}}$ and $\mathcal{S}_{\alpha_{k}}$, where $\beta_{j_{1}}, \beta_{j_{2}}, \ldots, \beta_{j_{h}}$ are the children of $\alpha_{k}$ in $\mathcal{F}$.

To establish the correctness of the confluent phase, we require a number of technical lemmas. The first one characterizes the spill events of basins on the flow path between a basin and its tributary. For three basins $\beta_{j}, \beta_{h} \in \mathcal{F}$ and a basin $\alpha \in\left\{\alpha_{i}, \beta_{i}\right\}$, we say $\beta_{h}$ is on the path from $\beta_{j}$ to $\alpha$ in $\mathcal{F}$ if $\beta_{h}$ is not a sub-basin of $\alpha$ but belongs to the path from $\beta_{j}$ to every sub-basin $\alpha^{\prime} \in \mathcal{F}$ of $\alpha$.

Lemma 3. Consider a tributary $\tau$ of a basin $\alpha \in\left\{\alpha_{i}, \beta_{i}\right\}$, and assume that $\tau \in\left\{\beta_{j}\right\} \cup \mathcal{S}_{\beta_{j}}$, for some $j$. Then $E_{\beta_{h}}$ is a watershed event, for every basin $\beta_{h}$ on the path from $\beta_{j}$ to $\alpha$ in $\mathcal{F}$ that is not a sub-basin of $\alpha_{i}$.

Proof. For $j>i$, the lemma holds vacuously because $\beta_{j}$ is a sub-basin of $\alpha_{i}$ in this case and $\alpha \in\left\{\alpha_{i}, \beta_{i}\right\}$; this implies that every basin $\beta_{h}$ on the path from $\beta_{j}$ to $\alpha$ is a sub-basin of $\alpha_{i}$. For $j \leq i$, assume there exists a basin $\beta_{h}$ on the path from $\beta_{j}$ to $\alpha$ that is not a sub-basin of $\alpha_{i}$ and such that $E_{\beta_{h}}$ is a basin event. Since $\beta_{h}$ is not a sub-basin of $\alpha_{i}$, we have $h \leq i$. If $\alpha=\alpha_{i}$, this implies that $\alpha$ is a sub-basin of $\alpha_{h}$ and $t_{\alpha} \leq t_{\alpha_{h}} \leq t_{\beta_{h}}$. If $\alpha=\beta_{i}$, then $h<i$ because $\beta_{h} \neq \alpha$. Thus, $\alpha$ is again a sub-basin of $\alpha_{h}$; in particular, $t_{\alpha} \leq t_{\beta_{h}}$. However, since $\beta_{j}$ is a tributary of $\alpha$, we have $t_{\beta_{j}}<t_{\alpha}$ and, therefore, $t_{\beta_{j}}<t_{\beta_{h}}$ in both cases. For $\beta_{j}$ to be a tributary of $\alpha$, there has to exist a down-hill path from $\beta_{j}$ to $\alpha$ at time $t_{\beta_{j}}$. By the choice of $\beta_{h}$, any path from $\beta_{j}$ to $\alpha$ has to pass through a point interior to $\beta_{h}$ first and then through $p_{\beta_{h}}$. Since $\beta_{h}$ is not full at time $t_{\beta_{j}}$, no such path is down-hill at time $t_{\beta_{j}}$, a contradiction.

Our next lemma shows that, if a basin $\tau \in\left\{\beta_{j}\right\} \cup \mathcal{R}_{\beta_{j}}$ is a tributary of a basin $\beta_{i}$ with $\beta_{j} \in \mathcal{F}_{\beta_{i}}$, then $\tau \in L_{\beta_{i}}$, that is, $\tau$ is processed when computing $t_{\beta_{i}}^{\prime}$; otherwise, if it is a tributary of $\alpha \in\left\{\alpha_{i}, \beta_{i}\right\}$, then it is in $L_{\gamma}$, for some sub-basin $\gamma$ of $\alpha_{i}$. The first part is used to prove in Lemma 5 below that the confluent phase computes the correct spill times for all confluent watershed events. The second part is used to establish the correctness of the diffluent phase, discussed in Section 3.3.3.

Lemma 4. Consider a basin $\tau \in\left\{\beta_{j}\right\} \cup \mathcal{S}_{\beta_{j}}$ with $\tau \in L_{\gamma}$. Assume further that every basin $\beta_{h}$ in $\mathcal{F}_{\gamma}$ satisfies $t_{\beta_{h}}^{\prime} \geq t_{\beta_{h}}$, with equality if $E_{\beta_{h}}$ is a watershed event. If $\tau$ is a tributary of $\beta_{i}$ and $\beta_{j} \in \mathcal{F}_{\beta_{i}}$, then $\gamma=\beta_{i}$. Otherwise, if $\tau$ is a tributary of a basin $\alpha \in\left\{\alpha_{i}, \beta_{i}\right\}$, then $\gamma$ is a sub-basin of $\alpha_{i}$.

Proof. First assume $\tau$ is a tributary of $\beta_{i}$ and $\beta_{j} \in \mathcal{F}_{\beta_{i}}$. Then $E_{\tau}$ is a watershed event and either $\tau \in \mathcal{S}_{\beta_{j}}$ or $\tau=\beta_{j}$. In both cases, we have $t_{\tau}^{\prime}=t_{\tau}$, in the latter case by the assumption that $t_{\beta_{h}}^{\prime}=t_{\beta_{h}}$ for every watershed event on the path from $\beta_{j}$ to $\gamma$. If $\gamma$ belongs to the path from $\beta_{j}$ to $\beta_{i}$ and $\gamma \neq \beta_{i}$, then we have $t_{\gamma}^{\prime}=t_{\gamma}<t_{\tau}$ because $\tau$ is a tributary of $\beta_{i}$ and, hence, $E_{\gamma}$ is a watershed event. However, $\gamma \neq \alpha_{k}$ in this case, and every element $\tau$ in $L_{\gamma}$ is removed from $Q_{\gamma}$ while computing $t_{\gamma}^{\prime}$. Thus, $t_{\tau}^{\prime}<t_{\gamma}^{\prime}$, a contradiction. This shows that $\gamma$ is an ancestor of $\beta_{i}$ in $\mathcal{F}$. This, however, implies that $t_{\beta_{i}}^{\prime} \geq t_{\beta_{i}}>t_{\tau}=t_{\tau}^{\prime}$. Thus, the computation of $t_{\beta_{i}}^{\prime}$ removes $\tau$ from $Q_{\beta_{i}}$, and $\tau \in L_{\beta_{i}}$. 
Now assume that $\tau$ is a tributary of a basin $\alpha \in\left\{\alpha_{i}, \beta_{i}\right\}$ and, if $\alpha=\beta_{i}$, that $\beta_{j} \notin \mathcal{F}_{\beta_{i}}$. Then, if $\gamma$ belongs to the path from $\beta_{j}$ to $\alpha_{i}$, we obtain $t_{\gamma}^{\prime}=t_{\gamma}<t_{\tau}=t_{\tau}^{\prime}$ on the one hand, because $\tau$ is a tributary of $\alpha$ and, by Lemma $3, E_{\gamma}$ is a watershed event; on the other hand, $t_{\gamma}^{\prime}>t_{\tau}^{\prime}$ because otherwise $\tau \notin L_{\gamma}$. Thus, we obtain a contradiction and $\gamma$ is a sub-basin of $\alpha_{i}$.

Lemma 5. For all $1 \leq i \leq k$, we have $t_{\beta_{i}}^{\prime} \geq t_{\beta_{i}}$. If $E_{\beta_{i}}$ is a watershed event, equality holds.

Proof. By induction on $\left|\mathcal{F}_{\beta_{i}}\right|$. The base case is $\left|\mathcal{F}_{\beta_{i}}\right|=0$, in which case there is nothing to prove. So consider a node $\beta_{i}$ and assume the claim holds for all its descendants. Then, by Lemma 4 , every tributary $\tau \in\left\{\beta_{j}\right\} \cup \mathcal{S}_{\beta_{j}}$, for some descendant $\beta_{j}$ of $\beta_{i}$, is inspected when computing $t_{\beta_{i}}^{\prime}$, and each such tributary satisfies $t_{\tau}^{\prime}=t_{\tau}$ by the inductive hypothesis. In particular, each such tributary belongs to $Q_{\beta_{i}}$ when invoking procedure FindSPILLTime to compute $t_{\beta_{i}}^{\prime}$. We prove that any other element $\alpha \in Q_{\beta_{i}}$ satisfies $t_{\alpha}^{\prime} \geq t_{\beta_{i}}$. By Lemma 2, this implies that $t_{\beta_{i}}^{\prime} \geq t_{\beta_{i}}$, with $t_{\beta_{i}}^{\prime}=t_{\beta_{i}}$ if $Q_{\beta_{i}}$ contains all tributaries of $\beta_{i}$, which is the case if $E_{\beta_{i}}$ is a watershed event. Indeed if there was a tributary $\tau$ such that $\tau \in\left\{\beta_{j}\right\} \cup \mathcal{R}_{\beta_{j}}$, for some $\beta_{j} \notin \mathcal{F}_{\beta_{i}}$, the water spilling from $\tau$ into $\beta_{i}$ would have to flow through a point interior to $\alpha_{i}$ and then through $p_{\alpha_{i}}=p_{\beta_{i}}$. Since $t_{\tau}<t_{\beta_{i}}$ and $E_{\beta_{i}}$ is a watershed event, $\alpha_{i}$ is not full at time $t_{\tau}$, that is, the path from $\tau$ to $\beta_{i}$ cannot be down-hill at time $t_{\tau}$, a contradiction.

So consider an element $\alpha \in Q_{\beta_{i}}$, and assume that $\alpha \in$ $\left\{\beta_{j}\right\} \cup \mathcal{S}_{\beta_{j}}$, for some $\beta_{j} \in \mathcal{F}_{\beta_{i}}$, and $t_{\alpha}^{\prime}<t_{\beta_{i}}$. We prove that $\alpha$ is a tributary of $\beta_{i}$. We have $t_{\alpha}^{\prime} \geq t_{\beta_{h}}^{\prime}$, for all $\beta_{h}$ on the path from $\beta_{j}$ to $\beta_{i}$, excluding $\beta_{i}$, because otherwise $\beta_{h}$ would have removed $\alpha$ from $Q_{\beta_{h}}$ when computing $t_{\beta_{h}}^{\prime}$. Since, by the inductive hypothesis, $t_{\beta_{h}} \leq t_{\beta_{h}}^{\prime}$, this implies that $t_{\beta_{h}}<t_{\beta_{i}}$. Also by the inductive hypothesis, we have $t_{\alpha}^{\prime} \geq t_{\alpha}$. If $t_{\alpha} \geq t_{\beta_{h}}$, for all $\beta_{h}$ on the path from $\beta_{j}$ to $\beta_{i}$, then $\alpha$ is a tributary of $\beta_{i}$. If $t_{\alpha}<t_{\beta_{h}} \leq t_{\alpha}^{\prime}$, for some $\beta_{h}$, then, by the inductive hypothesis, $E_{\alpha}$ is not a watershed event. In particular, $\alpha=\beta_{j}$, for some $j<i$, and $t_{\beta_{i}}<t_{\alpha_{j}} \leq$ $t_{\beta_{j}}=t_{\alpha}$ because $\beta_{i}$ is a sub-basin of $\alpha_{j}$. This contradicts our assumption that $t_{\alpha} \leq t_{\alpha}^{\prime}<t_{\beta_{i}}$.

\subsubsection{Diffluent Phase}

In the diffluent phase, we process the basins in $\mathcal{M}_{\beta}$ in the order $\alpha_{k}, \beta_{k}, \alpha_{k-1}, \beta_{k-1}, \ldots, \alpha_{1}, \beta_{1}$. We initialize a priority queue $Q$ to contain all events in $L_{\alpha_{k}}$. Then we repeat the following steps for $i=k, k-1, \ldots, 1$ :

First we compute $t_{\alpha_{i}}^{\prime \prime}$. If $i=k$, we do this by invoking procedure FINDSPILLTIME with arguments $\alpha_{k}, Q, u_{\alpha_{k}}:=0$, $W_{\alpha_{k}}:=W_{\alpha_{k}}^{0}$, and $V_{\alpha_{k}}^{r}:=V_{\alpha_{k}}$. If $i<k$, we invoke the procedure with arguments $\alpha_{i}, Q, u_{\alpha_{i}}:=\max \left(t_{\alpha_{i+1}}^{\prime \prime}, t_{\beta_{i+1}}^{\prime \prime}\right)$, $W_{\alpha_{i}}:=\max \left(W_{\alpha_{i+1}}, W_{\beta_{i+1}}\right)$, and $V_{\alpha_{i}}^{r}:=V_{\alpha_{i}}-V_{\alpha_{i+1}}-V_{\beta_{i+1}}$. We place the elements of $Q$ processed by procedure FindSpILLTime into a list $\mathcal{R}_{\alpha_{i}}^{\prime \prime}$.

Then we compute $t_{\beta_{i}}^{\prime \prime}$. To do so, we insert $\alpha_{i}$ (with priority $t_{\alpha_{i}}^{\prime \prime}$ ) and the events in $L_{\beta_{i}}$ into $Q$ and invoke procedure FindSPILLTIme with arguments $\beta_{i}, Q, u_{\beta_{i}}:=0$, $W_{\beta_{i}}:=W_{\beta_{i}}^{0}$, and $V_{\beta_{i}}^{r}:=V_{\beta_{i}}$. We place the elements of $Q$ processed by procedure FindSPILLTIME into a list $\mathcal{R}_{\beta_{i}}^{\prime \prime}$.

The computation of $t_{\alpha_{i}}^{\prime \prime}$ and $t_{\beta_{i}}^{\prime \prime}$ may not process all elements in $Q$, and some of these elements may be sub-basins of $\alpha_{i}$. These elements should be ignored in subsequent computations, as they cannot be tributaries of any $\alpha_{j}$ or $\beta_{j}$ with $j<i$. To ensure this, we augment the above procedure to ignore in iteration $i$ all elements $\alpha_{j}$ or $\beta_{j}$ in $Q$ with $j>i$. (Note that we ignore only these basins, not the elements of $\mathcal{S}_{\alpha_{k}}$ or $\mathcal{S}_{\beta_{j}}$ with $j>i$.)

LeMma 6. For all $1 \leq i \leq k$, we have $t_{\alpha_{i}}=t_{\alpha_{i}}^{\prime \prime}, t_{\beta_{i}}=t_{\beta_{i}}^{\prime \prime}$, $\mathcal{R}_{\beta_{i}}=\mathcal{R}_{\beta_{i}}^{\prime \prime}$, and $\mathcal{R}_{\alpha_{i}}=\mathcal{R}_{\alpha_{k}}^{\prime \prime} \cup \bigcup_{j=i}^{k-1} \mathcal{R}_{\beta_{j}}^{\prime \prime}$.

Proof. By induction on $i$. For $i=k$, Lemmas 4 and 5 imply that every tributary $\tau$ of $\alpha_{k}$ belongs to $L_{\alpha_{k}}$ and satisfies $t_{\tau}^{\prime}=t_{\tau}$. Any other basin $\alpha \in L_{\alpha_{k}}$ satisfies $t_{\alpha}^{\prime} \geq t_{\alpha}$, and the same arguments as in the proof of Lemma 5 show that $t_{\alpha}^{\prime} \geq t_{\alpha_{k}}$ in this case. Hence, by Lemma 2 , the first iteration computes $t_{\alpha_{k}}^{\prime \prime}=t_{\alpha_{k}}$ and processes only tributaries of $\alpha_{k}$, that is, $\mathcal{R}_{\alpha_{k}}^{\prime \prime}=\mathcal{R}_{\alpha_{k}}$.

Now consider $\beta_{k}$. By Lemmas 4 and 5 , every tributary of $\beta_{k}$ belongs to $\left\{\alpha_{k}\right\} \cup L_{\alpha_{k}} \cup L_{\beta_{k}}$, and we have just argued that the computation of $\alpha_{k}$ processes only tributaries of $\alpha_{k}$. Hence, $Q$ contains all tributaries of $\beta_{k}$. We have just argued that $t_{\alpha_{k}}^{\prime \prime}=t_{\alpha_{k}}$ and, by Lemma 5 , any other tributary $\tau$ of $\beta_{k}$ satisfies $t_{\tau}^{\prime}=t_{\tau}$. For an element $\alpha \in Q$ that is not a tributary of $\beta_{k}$, the same arguments as in the proof of Lemma 5 show that $t_{\alpha}^{\prime} \geq t_{\beta_{k}}$. (However, we have to distinguish the cases $\beta_{j} \in \mathcal{F}_{\beta_{k}}$ and $\beta_{j} \notin \mathcal{F}_{\beta_{k}}$, where $\alpha \in\left\{\beta_{j}\right\} \cup \mathcal{R}_{\beta_{j}}$.) Thus, by Lemma 2 , we compute $t_{\beta_{k}}^{\prime \prime}=t_{\beta_{k}}$ and $\mathcal{R}_{\beta_{k}}^{\prime \prime}=\mathcal{R}_{\beta_{k}}$.

Now assume that $i<k$ and that the inductive hypothesis holds for all $j>i$. By Lemmas 4 and 5, every tributary $\tau$ of $\alpha_{i}$ is contained in $L:=L_{\alpha_{k}} \cup \bigcup_{j=i+1}^{k} L_{\beta_{j}}=$ $Q \cup \bigcup_{j=i+1}^{k}\left(\mathcal{R}_{\alpha_{j}}^{\prime \prime} \cup \mathcal{R}_{\beta_{j}}^{\prime \prime}\right)$ and satisfies $t_{\tau}^{\prime}=t_{\tau}$. Using the arguments from the proof of Lemma 5 again, every $\alpha \in L$ that is not a tributary of $\alpha_{i}$ satisfies $t_{\alpha}^{\prime} \geq t_{\alpha_{i}}$. Moreover, the elements of $L \backslash Q$ are exactly the tributaries of $\alpha_{i+1}$ and $\beta_{i+1}$, that is, the tributaries $\tau$ of $\alpha_{i}$ that satisfy $t_{\tau}<u_{\alpha_{i}}$. Hence, by Lemma 2, we compute $t_{\alpha_{i}}^{\prime \prime}=t_{\alpha_{i}}$ and $\mathcal{R}_{\alpha_{i}}^{\prime \prime}$ to contain exactly the tributaries $\tau$ of $\alpha_{i}$ that satisfy $t_{\tau} \geq u_{\alpha_{i}}$. Thus, $\mathcal{R}_{\alpha}=\mathcal{R}_{\alpha_{i}}^{\prime \prime} \cup \bigcup_{j=i+1}^{k}\left(\mathcal{R}_{\alpha_{j}}^{\prime \prime} \cup \mathcal{R}_{\beta_{j}}^{\prime \prime}\right)$. The correctness proof for the computation of $t_{\beta_{i}}^{\prime \prime}$ and $\mathcal{R}_{\beta_{i}}^{\prime \prime}$ is identical to that for $\beta_{k}$.

Lemma 7. The computation of the spill times $t_{\alpha_{i}}$ and $t_{\beta_{i}}$ and of the tributary lists $\mathcal{R}_{\beta_{i}}$, for all $1 \leq i \leq k$, takes $\mathrm{O}(\operatorname{sort}(X)) I / O s$.

PROOF. The correctness of the algorithm is established by Lemma 6 . Its I/O-complexity is established as follows: Both phases of the algorithm perform $\mathrm{O}(X)$ priority queue operations, as every event is inserted and removed from a priority queue only once in each of the two phases and the confluent phase performs at most $X$ MELD operations. By Theorem 3 in Section 4, these priority queue operations cost $\mathrm{O}(\operatorname{sort}(X)) \mathrm{I} /$ Os. Apart from this, the algorithm traverses trees $\mathcal{F}$ and $\mathcal{M}(S)$ once each and scans lists associated with the tree nodes of total length $\mathrm{O}(X)$. After arranging the tree nodes and list elements in the right order, using a sorting step, the scanning of these lists takes $\mathrm{O}(X / B) \mathrm{I} / \mathrm{Os}$.

\subsection{Computing the Flooding Times of All Ter- rain Vertices}

The terrain vertices that are not spill points of basins can be seen as partitioning the basins of $\mathcal{T}$ into sub-basins as follows. For each terrain vertex $v$, let $\alpha_{v}$ be the smallest basin that contains $v$. For a basin $\beta$, let $V(\beta)$ be the set of terrain vertices with $\alpha_{v}=\beta$. Each vertex $v \in V(\beta)$ defines a sub-basin $\beta_{\mathcal{T}(v)}$ of $\beta$ containing the portion of $\beta$ below elevation $\mathcal{T}(v)$. Let $v_{1}, v_{2}, \ldots, v_{k}$ be the vertices in $V(\beta)$ sorted by 
increasing elevation. Then the water from every tributary in $\mathcal{R}_{\beta_{i}}^{\prime \prime}$ first collects in $\beta_{\mathcal{T}\left(v_{1}\right)}$; once $\beta_{\mathcal{T}\left(v_{1}\right)}$ is full, the water collects in $\beta_{\mathcal{T}\left(v_{2}\right)}$, and so on. This is just a simplified version of the diffluent flow computation in Section 3.3.3, and computing the spill times of basins $\beta_{\mathcal{T}\left(v_{1}\right)}, \beta_{\mathcal{T}\left(v_{2}\right)}, \ldots, \beta_{\mathcal{T}\left(v_{k}\right)}$ - that is, the flooding times of vertices $v_{1}, v_{2}, \ldots, v_{k}$-from the list $\mathcal{R}_{\beta}^{\prime \prime}$ takes $\mathrm{O}(\operatorname{sort}(N)) \mathrm{I} / \mathrm{Os}$ in total for all basins of $\mathcal{T}$. The computation of the sets $V(\beta)$ can be incorporated in the computation of the basins of $\mathcal{T}$ without increasing the I/O complexity of that phase. Details appear in the full paper. Thus, we have the following result.

THEOREM 2. The flooding times of all vertices of a terrain $\mathcal{T}$ with $N$ vertices and $X$ pits can be computed using $\mathrm{O}(\operatorname{sort}(X) \log (X / M)+\operatorname{sort}(N)) I / O s$, given the watersheds and volumes of all basins of $\mathcal{T}$.

\section{A MELDABLE PRIORITY QUEUE}

In this section, we discuss how to extend the external heap of Fadel et al. [12] to obtain a meldable priority queue that can be used in the procedure in Section 3.3.2. This structure maintains a sequence of priority queues $Q_{1}, Q_{2}, \ldots, Q_{k}$ under Create, Insert, DeleteMin, and Meld operations. Given the current sequence $Q_{1}, Q_{2}, \ldots, Q_{k}$, a CREATE operation creates a new, empty priority queue $Q_{k+1}$ and appends it to the end of the sequence; an $\operatorname{INSERT}(x)$ operation inserts element $x$ into $Q_{k}$; a DeleteMin operation deletes and returns the minimum element in $Q_{k}$; a MELD operation replaces $Q_{k-1}$ and $Q_{k}$ with a new priority queue $Q_{k-1}^{\prime}:=Q_{k-1} \cup Q_{k}$ containing the elements from $Q_{k-1}$ and $Q_{k}$. We prove the following result.

TheOREM 3. There exists a linear-space data structure that uses $\mathrm{O}(\operatorname{sort}(N)) I / O$ s to process a sequence of $N$ CREATE, Insert, DeleteMin, and Meld operations.

\subsection{The Structure}

Each priority queue $Q_{i}$ is represented as an external heap similar to the one presented in [12]. The underlying structure is a rooted tree whose internal nodes have between $a=M /(4 B)$ and $b=M / B$ children. There are two types of leaves: regular leaves are on the lowest level of the tree, which we call the leaf level; leaves above the leaf level are special.

Every node $v$ has an associated buffer $X_{v}$. If $v$ is an internal node, $X_{v}$ has size $M$. If $v$ is a leaf, there is no bound on the size of $X_{v}$. The elements in $X_{v}$ are sorted, and the buffer contents of adjacent nodes satisfy the heap property: for every node $v$ with parent $u$ and every pair of elements $x \in X_{u}$ and $y \in X_{v}$, we have $x \leq y$.

To support INSERT and DELETEMin operations efficiently, every priority queue $Q_{i}$ is equipped with an insert/delete buffer or $I / D$ buffer for short. This buffer is capable of holding up to $M$ elements and stores the minimum elements in $Q_{i}$ as well as newly inserted elements. To distinguish newly inserted elements in $Q_{i}$ 's I/D buffer from minimum elements, $Q_{i}$ has an associated priority $p_{i}$, which is the minimum priority of the elements stored in the external part of $Q_{i}$.

The I/D buffers of priority queues $Q_{1}, Q_{2}, \ldots, Q_{k}$ are kept on a buffer stack, with the buffer of $Q_{1}$ at the bottom and the buffer of $Q_{k}$ at the top. The I/D buffers of consecutive priority queues are separated by special marker elements.
We always keep the topmost $M$ elements of this stack in memory, which ensures in particular that the I/D buffer of $Q_{k}$ is in memory.

\subsection{Operations}

Create. To create a new priority queue $Q_{k+1}$, a CREATE operation pushes a new marker element onto the buffer stack.

Insert. An INSERT operation on $Q_{k}$ adds the inserted element $x$ to $Q_{k}$ 's I/D buffer. If the buffer now contains $M$ elements, we FLusH the buffer as described below. Then we FILL the I/D buffer with the $M / 2$ minimum elements in $Q_{k}$ and update $p_{k}$ accordingly.

DeleteMin. A DeleteMin operation on $Q_{k}$ returns the minimum element in $Q_{k}$ 's I/D buffer. Before doing so, however, it checks whether the I/D buffer of $Q_{k}$ is empty or its minimum element is greater than $p_{k}$. If so, it FLusHes the I/D buffer and then Fills it with the $M / 2$ minimum elements in $Q_{k}$.

Meld. A MELD operation behaves differently depending on the structure of the two involved priority queues, $Q_{k-1}$ and $Q_{k}$. During different stages of its life time, a priority queue may have no external portion because all its elements fit in the I/D buffer. If at least one of the two priority queues, say $Q_{k}$, has no external portion, we destroy its I/D buffer, and INSERT its elements into $Q_{k-1}$. If both priority queues have external portions, we FLUSH and destroy their I/D buffers, MERGE their two trees as described below, and then FILL the I/D buffer of the merged priority queue $Q_{k-1}^{\prime}:=Q_{k-1} \cup Q_{k}$ with the $M / 2$ minimum elements in $Q_{k-1}^{\prime}$.

Flush. A FLUSH operation of an I/D buffer containing $K$ elements creates a new leaf $l$ at the leaf level and stores these $K$ elements in $l$. Then it applies a HEAPIFY operation to the path from $l$ to the root to restore the heap property. If the addition of $l$ increased the degree of $l$ 's parent $p$ to $M / B+1$, we split $p$ into two nodes $p^{\prime}$ and $p^{\prime \prime}$, each with half of $p$ 's children. We associate the buffer of $p$ with $p^{\prime}$ and populate the buffer of $p^{\prime \prime}$ with the $M$ smallest elements in its subtree using a FILL operation. If this split increases the degree of $p$ 's parent to $M / B+1$, we apply this rebalancing procedure recursively until we reach the root. If the root has degree greater than $M / B$, we split it into two nodes with a new parent.

Fill. A FILL operation applied to a node $v$ repeatedly takes the smallest element stored in the buffers of $v$ 's children, removes it from the corresponding child's buffer and stores it in $v$ 's buffer. This continues until $M$ elements have been collected in $v$ 's buffer or there are no elements left in the buffers of $v$ 's children. Whenever a child buffer runs empty while filling $v$ 's buffer, its buffer is filled recursively before continuing to fill $v$ 's buffer. Once there are no more elements left in a node $v$ 's subtree, we mark $v$ as exhausted, in order to avoid repeatedly trying to fill $v$ with elements. More precisely, a node is marked as exhausted once its buffer becomes empty and all its children are exhausted. To fill the I/D buffer of a priority queue $Q_{k}$ with the $M / 2$ smallest elements in $Q_{k}$, we transfer the $M / 2$ smallest elements from the root of $Q_{k}$ into the I/D buffer, FILLing the root's buffer whenever it runs empty. If the root becomes exhausted in the process, we delete the entire external portion of the priority queue. 
Merge. A Merge operation between two trees $T_{1}$ and $T_{2}$ of heights $h_{1}>h_{2}$ proceeds as follows. We locate a node $v$ at height $h_{2}+1$ in $T_{1}$ and make the root $r_{2}$ of $T_{2}$ a child of $v$. We also create a new special leaf node $l$ that is a child of $v$. Now let $v=v_{0}, v_{1}, \ldots, v_{h}$ be the ancestors of $v$, by increasing distance from $v$, let $K_{i}$ be the number of elements in $X_{v_{i}}$, and let $K=\sum_{i=0}^{h} K_{i}$. First we collect the elements in $X_{v_{0}}, X_{v_{1}}, \ldots, X_{v_{h}}$ in sorted order and store them in $l$. Then we repeatedly remove the minimum element from $X_{r_{2}}$ and $X_{l}$, refilling $X_{r_{2}}$ as necessary, until we have collected the $K$ smallest elements in the subtrees rooted at $r_{2}$ and $l$. We store these elements in buffers $X_{v_{0}}, X_{v_{1}}, \ldots, X_{v_{h}}$, sorted top-down and so that each node $v_{i}$ receives $K_{i}$ elements. We clear the "exhausted" labels of all nodes among $v_{0}, v_{1}, \ldots, v_{h}$. If $v$ has degree greater than $M / 2$ as a result of gaining two children, $r_{2}$ and $l$, we rebalance the tree using node splits starting at $v$ similar to the rebalancing done after a FLUSH operation.

Heapify. The final operation to discuss is the HEAPIFY operation. Given a node $v$ and its path $v=v_{0}, v_{1}, \ldots, v_{h}$ to the root, a HEAPIFY operation ensures that the elements stored on the path satisfy the heap property. It assumes that the elements in $v_{1}, v_{2}, \ldots, v_{h}$ already do so, but some elements in $v_{0}$ may be less than elements stored at higher nodes. To restore the heap property, we sort the elements in $v_{0}$ and collect the elements in $v_{1}, v_{2}, \ldots, v_{h}$ in sorted order. Then we merge the two sorted sequences to obtain a sorted sequence of the elements stored in $v_{0}, v_{1}, \ldots, v_{h}$ and distribute these elements over the buffers of nodes $v_{0}, v_{1}, \ldots, v_{h}$, assigning the same number of elements to each node as it had before the operation and storing the elements sorted top-down on the path. If some of the nodes on the path were marked as exhausted before this operation, we unmark them.

\subsection{Analysis}

To prove the correctness of all priority queue operations, we need to verify that the heap property is maintained at all times. This is little more than an exercise and is therefore omitted.

It is easy to see that every Create, Insert, DeleteMin, and MELD operation makes only $\mathrm{O}(1)$ changes to the buffer stack and thus has an amortized cost of $\mathrm{O}(1 / B) \mathrm{I} / \mathrm{Os}$, excluding the manipulations performed by the FLUSH, FILL, Merge, and Heapify operations they trigger. The following two lemmas bound the cost of all Flush, Fill, Merge, and HEAPIFY operations performed during a sequence of $N$ priority queue operations. Due to lack of space, their proofs are omitted.

LEMMA 8. During a sequence of $N$ priority queue operations, at most $\mathrm{O}(N / M)$ Flush, FILL, MERgE, and HeAPIFY operations are performed.

Lemma 9. The amortized cost per Flush, Fill, Merge or HEAPIFY operation is $\mathrm{O}\left((M / B) \log _{M / B}(N / M)\right) I / O s$.

\section{REFERENCES}

[1] P. Agarwal, L. Arge, and K. Yi. I/O-efficient batched union-find and its applications to terrain analysis. In Proc. 22nd SCG, pp. 167-176, 2006.

[2] A. Aggarwal and J. S. Vitter. The input/output complexity of sorting and related problems. Comm. of the ACM, 31(9):1116-1127, 1988.
[3] L. Arge. The buffer tree: A technique for designing batched external data structures. Algorithmica, 37(1):1-24, 2003.

[4] L. Arge, J. Chase, P. Halpin, L. Toma, D. Urban, J. S. Vitter, and R. W. and. Flow computation on massive grid terrains. In Proc. 9th ACM GIS, pp. 82-87, 2001.

[5] L. Arge and M. Revsbæk. I/O-efficient contour tree simplification. In Proc. 20th ISAAC, pp. 1155-1165, 2009.

[6] Y.-J. Chiang, M. T. Goodrich, E. F. Grove, R. Tamassia, D. E. Vengroff, and J. S. Vitter. External-memory graph algorithms. In Proc. 6th SODA, pp. 139-149, 1995.

[7] A. Danner. I/O Efficient Algorithms and Applications in Geographic Information Systems. PhD thesis, Dept. of Comp. Sci., Duke University, 2006.

[8] A. Danner, K. Yi, T. Mølhave, P. K. Agarwal, L. Arge, and H. Mitasova. TerraStream: From elevation data to watershed hierarchies. Manuscript, 2007.

[9] M. de Berg, O. Cheong, H. Haverkort, J.-G. Lim, and L. Toma. I/O-efficient flow modelling on fat terrains. In Proc. 10th WADS, pp. 239-250, 2007.

[10] H. Edelsbrunner, J. Harer, and A. Zomorodian. Hierarchical morse complexes for piecewise linear 2-manifolds. In Proc. 17th SCG, pp. 70-79, 2001.

[11] H. Edelsbrunner, D. Letscher, and A. Zomorodian. Topological persistence and simplification. In Proc. 41st FOCS, pp. 454-463, 2000.

[12] R. Fadel, K. V. Jakobsen, J. Katajainen, and J. Teuhola. Heaps and heapsort on secondary storage. Theor. Comp. Sci., 220(2):345-362, 1999.

[13] S. Jenson and J. Domingue. Extracting topographic structure from digital elevation data for geographic information system analysis. Photogrammetric Eng. and Remote Sensing, 54(11):1593-1600, 1988.

[14] Y. Liu and J. Snoeyink. Flooding triangulated terrain. In Proceedings of the 11th International Symposium on Spatial Data Handling, pages 137-148, 2005.

[15] J. F. O'Callaghan and D. M. Mark. The extraction of drainage networks from digital elevation data. Computer Vision, Graphics and Image Proc., 28(3):323-344, 1984.

[16] O. Palacios-Velez, W. Gandoy-Bernasconi, and B. Cuevas-Renaud. Geometric analysis of surface runoff and the computation order of unit elements in distributed hydrological models. J. Hydrology, 211:266-274, 1998.

[17] D. M. Theobald and M. F. Goodchild. Artifacts of TIN-based surface flow modeling. In Proc. GIS/LIS'90, pp. 955-964, 1990.

[18] G. Tucker, S. Lancaster, N. Gasparini, and S. Rybarczyk. An object-oriented framework for hydrology and geomorphic modeling using triangulated irregular networks. Computers and Geosciences, 27(8):959-973, 2001. 